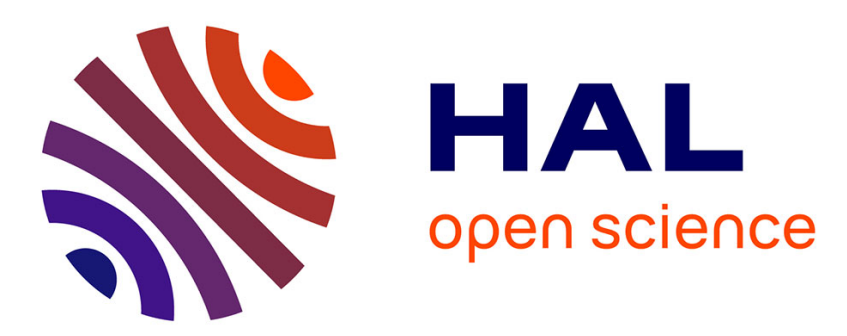

\title{
Energy losses in interacting fine-particle magnetic composites
}

\author{
F Burrows, C Parker, R F L Evans, Y Hancock, O Hovorka, R W Chantrell
}

\section{To cite this version:}

F Burrows, C Parker, R F L Evans, Y Hancock, O Hovorka, et al.. Energy losses in interacting fine-particle magnetic composites. Journal of Physics D: Applied Physics, 2010, 43 (47), pp.474010. 10.1088/0022-3727/43/47/474010.xml . hal-00570718

\section{HAL Id: hal-00570718 \\ https://hal.science/hal-00570718}

Submitted on 1 Mar 2011

HAL is a multi-disciplinary open access archive for the deposit and dissemination of scientific research documents, whether they are published or not. The documents may come from teaching and research institutions in France or abroad, or from public or private research centers.
L'archive ouverte pluridisciplinaire HAL, est destinée au dépôt et à la diffusion de documents scientifiques de niveau recherche, publiés ou non, émanant des établissements d'enseignement et de recherche français ou étrangers, des laboratoires publics ou privés. 


\title{
Energy losses in interacting fine-particle magnetic composites.
}

\author{
F. Burrows ${ }^{1}$, C. Parker ${ }^{1}$, R.F.L. Evans ${ }^{1}$, Y. Hancock $^{1} \ddagger$, O. \\ Hovorka $^{2}$ and R.W. Chantrell ${ }^{1}$ \\ ${ }^{1}$ Department of Physics, The University of York, Heslington Road, York, YO50 5DD, \\ U.K. \\ ${ }^{2}$ CIC nanoGUNE Consolider, Donostia, San Sebastian 20009, Spain. \\ E-mail: yh546@york.ac.uk
}

\begin{abstract}
The coercivity and energy losses in superparamagnetic magnetite and FePt nanoparticle composites subjected to an external, alternating magnetic field have been calculated as a function of the mean particle-size and packing density. The effect of interactions has been investigated by fitting the Sharrock law to the coercivity results as a function of the field cycle frequency of the magnetic field. This fitting leads to effective parameters for the anisotropy field $H_{K}^{\text {eff }}$ and $\beta^{\text {eff }}=K V / k_{B} T$, which are themselves dependent on the interaction strength. The increase or decrease of the coercivity with interactions depends upon the relative change of $H_{K}^{e f f}$ and $\beta^{e f f}$, thus demonstrating the complex effect that interactions have in these nanoparticle composites. The interparticle interactions have a non-trivial effect on the energy loss per cycle. The energy loss is reduced for systems with larger particles since the reduction in coercivity together with a corresponding reduction in the remanence dominates. For small particle sizes, the energy loss is increased. The primary mechanism here seems to be an enhancement of the energy barrier due to interactions, which changes the nature of the particles from superparamagnetic to being thermally stable.
\end{abstract}

PACS numbers: 75.40.Mg, 75.50.Tt, 75.60.Ej, 75.75.-c 


\section{Introduction}

Hyperthermia has been shown to be a promising adjuvant cancer treatment, which in combination with standard cancer treatments, such as radiotherapy and chemotherapy, increases both the control of the disease and also the overall patient survival rate [1][4]. Medical hyperthermia involves heat treatment of malignancies, which can be applied both locally to the tumour, or to the whole body. For whole body hyperthermia, the relative toxicity of normal tissue compared to tumour tissue is an important factor. Therefore, to minimise the risk of toxicity, chemotherapy or radiotherapy with localized hyperthermia would seem to offer the better option for a safe and effective treatment $[1,3]$.

A recently developed method of local hyperthermia is magnetic fluid hyperthermia, which involves the injection of magnetic nanoparticles that are suspended in a fluid either intravenously, or directly into the tumour site [5]. Once the magnetic nanoparticles are in place at the tumour site, an alternating magnetic field is then applied to the local area. Through hysteresis loop sweeping, energy is then dissipated from the magnetic nanoparticles into the tumour site, thereby heating the local area and destroying the tumour cells.

A number of theoretical approaches, for example Ref. [6], focus on the intrinsic losses in superparamagnetic particles, often considered in colloidal form. However, it should also be possible to generate heat via hysteresis losses in ferromagnetically stable particles, especially if they were deposited from the ferrofluid in a higher density non-colloidal state [7]. Eddy current losses are also possible, but should be a small contribution for particles in the $10 \mathrm{~nm}$ size range as determined by R. Ramprasad et. al [8]. In practice, probably both mechanisms contribute, but no overall model currently exists. Here, we focus on the hysterestic mechanism, specifically taking account of the interparticle interactions. Because of the possibility of non-colloidal states we study and require packing densities beyond that achievable for a ferrofluid.

The strength and frequency of the applied ac magnetic field are important considerations in local hyperthermia treatment. Using a stronger field, or increasing the frequency, should both increase the heat output, however, there are limitations as to what can be safely tolerated by the human body. The frequency and the product of the frequency and the magnetic field strength should be such that $f_{\max }<1.2 \mathrm{MHz}$ and $H_{\text {max }} f<6.10 \times 10^{6} \mathrm{Oes}^{-1}$, otherwise stimulation of the patient's nerves will cause pain $[4]$.

The type of magnetic material that is selected for hyperthermia treatment is also an important consideration, as more heat will be generated by a material that has a greater magnetic anisotropy and/or saturation magnetisation. In addition, there are many magnetic materials that are highly toxic to the human body. Magnetite is one magnetic material that has been proven to be biocompatible [9]. Another possibility is to use a stronger magnetic material, for example, Fe or FePt, that has been coated with an inert, biocompatible substance such as gold $[10,11]$. In this work, we consider 
both magnetite and FePt nanoparticle composites as candidate systems in a model study of hyperthermia treatment. Specifically, we investigate the role of interactions in determining the magnetic hysteresis properties and energy losses of these systems, therefore exploring their potential for hyperthermia applications.

The paper is organised as follows. First we describe the basic physics of the dynamic magnetic properties of the magnetite and FePt composite systems, taking into account the magnetostatic interactions, which might be of particular importance for high-moment materials. Next we consider the hysteresis losses as a function of the frequency, concluding with a calculation of hysteresis loss, which takes into account the maximum available field as a function of frequency.

\section{Theoretical Method}

The superparamagnetic (SPM) nanoparticle composite is modelled using a kinetic Monte-Carlo (MC) approach, which takes into account the behaviour of both the thermally stable and SPM particles, and is described in detail in [12]. We define the composite system as a cubic cell of interacting single-domain nanoparticles, with the particle sizes and the anisotropy fields generated according to a log-normal distribution function, and the value of the anisotropy constant $(\mathrm{K})$ having a 0.1 standard deviation. A random three-dimensional distribution of the anisotropy easy-axes is also used, thereby making the composite comparable to a granular system.

Interactions between the particles, which are responsible for coupling the SPM and thermally stable particle fractions, have been included via the calculation of a local field. The local field acts on each particle $i$ and is the vector sum of the dipolar interaction field produced by the neighbouring particles $j$ and the applied field $\boldsymbol{H}_{\boldsymbol{a p p}}$, such that,

$$
\boldsymbol{H}_{\text {loc }}=\sum_{j \neq i} \frac{3\left(\boldsymbol{\mu}_{\boldsymbol{j}} \cdot \boldsymbol{r}_{\boldsymbol{i j}}\right)}{r_{i j}^{5}}-\frac{\boldsymbol{\mu}_{\boldsymbol{j}}}{r_{i j}^{3}}+\boldsymbol{H}_{\boldsymbol{a p p}}
$$

Here, the summation is carried out over all particles within a range $r<r_{\max }$, where $r_{\max }$ is five times the median diameter. Contributions from particles outside of this range have been calculated using a mean-field approximation. This depends on the sample shape, which have assumed is spherical.

We define a thermally stable particle as one that satisfies $K V>k_{B} T \ln \left(t_{m} f_{0}\right)$, where $V$ is the volume of the particle, $k_{B}$ is the Boltzmann constant, $T$ is the temperature, $t_{m}$ is the measurement time and $f_{0}=10^{-9} s^{-1}$ is the attempt frequency. The equilibrium position of the moment of such a particle in the local field is calculated using the Stoner-Wohlfarth (SW) model [16]. If the SW model gives two equilibrium positions in the energy landscape, the moment can then jump between these positions with a reversal probability,

$$
P_{r}=1-\exp \left(t_{m} / \tau\right)
$$

that is given by the Arrhenius-Néel law [17], where $\tau$ is the relaxation time, which depends on the $T, V$ and $K$ of the particle. The energy barrier to reversal, $\Delta E\left(\psi, \boldsymbol{H}_{\boldsymbol{l o c}}\right)$, 
where $\psi$ is the orientation of the easy-axis relative to the local field, is calculated using a numerical approximation due to Pfeiffer [18], such that,

$$
\Delta E\left(\psi, \boldsymbol{H}_{\text {loc }}\right)=K V\left[1-\left|\boldsymbol{H}_{\text {loc }}\right| / g(\psi)\right]^{\kappa(\psi)},
$$

with $g(\psi)=\left[\cos ^{2 / 3} \psi+\sin ^{2 / 3} \psi\right]^{-3 / 2}$, and $\kappa(\psi)=0.86+1.14 g(\psi)$. Note that the local field $\boldsymbol{H}_{\boldsymbol{l o c}}$ is dependent on the interparticle interactions, which thus have an effect on the energy barrier and hence the dynamic properties.

SPM behaviour occurs up to large energy barriers, which for a measurement time of 100 s can typically be up to $25 k_{B} T$. Persistence in the SPM behaviour creates difficulties for standard $\mathrm{MC}$ approaches due to the unreasonably large number of $\mathrm{MC}$ steps that are necessary to achieve equilibrium. By considering the SPM particles with large energy barriers $\left(>3 k_{B} T\right)$ as a two-state system, an improved computational approach can therefore be derived [12]. This approach leads to the condition that, if the reversal transition is allowed, the moment is then assigned to either energy minimum with a probability,

$$
p=e^{-E_{i}} /\left(e^{-E_{1}}+e^{-E_{2}}\right),
$$

with $i=1,2$ labelling the minima. This condition ensures that the population of the two states obeys the Boltzmann distribution in thermal equilibrium [12]. For smaller energy barriers $\left(\Delta E<3 k_{B} T\right)$, a standard Metropolis algorithm is used, with the angles of the magnetic moment $\theta$ and $\phi$ being modified randomly [19]. In this case, the energy difference $\Delta E$ between the new energy state and the previous one is calculated and the moment is then allowed to remain in its new position with the probability $p=\min \left(1, e^{-\Delta E / k_{B} T}\right)$. For thermally stable particles (after determining the relevant minimum), we use standard MC moves to model the thermal equilibrium distribution about the energy minimum.

The model described here has been previously applied to a cubic computational cell in which particles have been placed at random [12]. This approach, however, fails for high densities of particles due to an increasing probability of overlap with an existing particle in the computational cell. To resolve this problem, a computational approach has been developed whereby an initial low density configuration (obtained by random placement of the particles) is compressed isothermally to the required density. The particles are given a log normal size distribution with a standard deviation of 0.1 [13]. The compression algorithm is defined by the following steps:

(i) Each particle is assigned a volume according to a lognormal distribution.

(ii) The particle is placed randomly in the cubic computational cell at low density.

(iii) The cell is shrunk until 2 particles touch.

(iv) $100 \mathrm{MC}$ moves are carried out to equilibrate the system.

(v) Steps 3 and 4 are repeated until the required packing density is reached.

(vi) $100 \mathrm{MC}$ moves are carried out to equilibrate the final configuration. 
This algorithm efficiently produces configurations for particle packing densities up to $50 \%$ that of the composite cell volume. The configurations that are generated are then used as inputs into the hysteresis loop model, and allow the study of the effects of interactions as a function of the particle packing density.

\section{Results and Discussion}

The model described in Section 2 is used to study the effects of interparticle magnetostatic interactions on the dynamic properties, and in particular, the energy losses as a function of the field cycle frequency for an SPM fine-particle composite consisting of magnetite and FePt. The interaction effects are investigated by varying the packing fraction of the composite systems. We assume a temperature $T=310 \mathrm{~K}$, and values of $\mathrm{K}$ and the saturation magnetisation $\left(M_{S}\right)$ of $5 \times 10^{6} \mathrm{erg} / \mathrm{cc}[14]$ and $1700 \mathrm{emu} / \mathrm{cc}$ for $\mathrm{FePt}$ and $5 \times 10^{5} \mathrm{erg} / \mathrm{cc}$ [15] and $400 \mathrm{emu} / \mathrm{cc}$ for magnetite, respectively.

\subsection{Sweep rate dependence of magnetic properties}

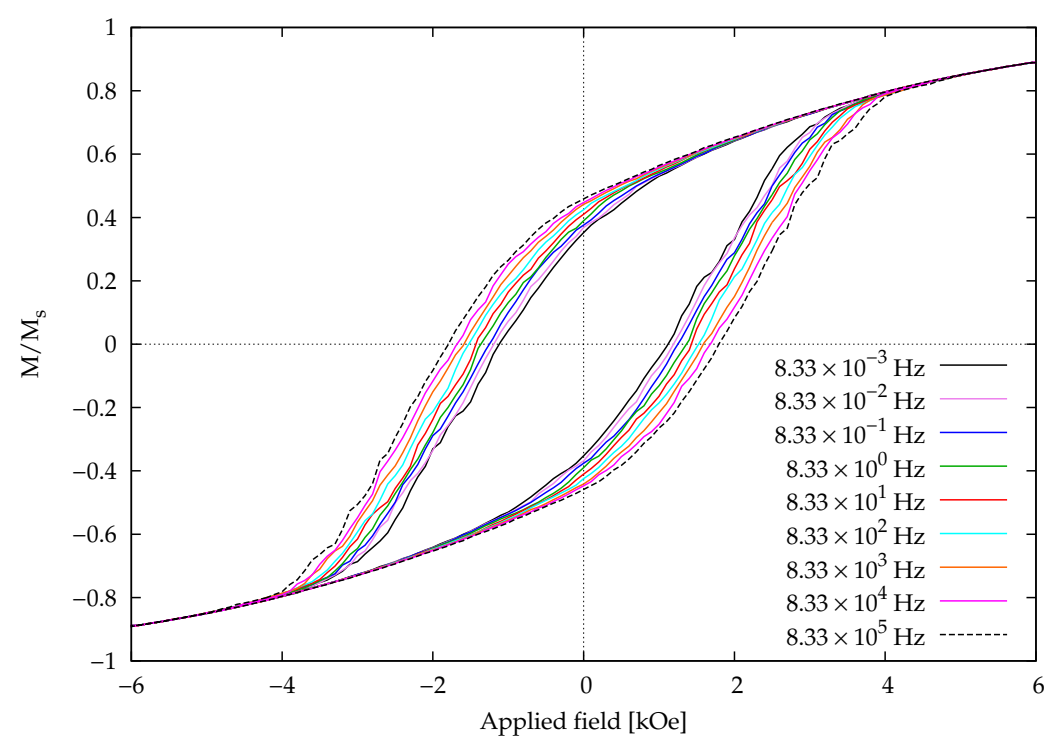

Figure 1: Magnetisation curves for various field cycle frequencies for an FePt nanoparticle composite with $5 \%$ packing density. The magnetisation curves are consistent with that which is expected for SW particles with randomly orientated easy-axes, namely, the coercivity and remanence increases as a function of the increasing field cycle frequency (hence sweep rate) [16].

A series of magnetisation curves for FePt particles as a function of the field cycle frequency is shown in Fig. 1. The particle packing density is low (5\% that of the composite cell volume), hence the interactions are relatively weak. The magnetisation curves have the form expected for a system consisting of SW particles with randomly oriented easy-axes in that the coercivity $H_{c}$ increases as a function of the field cycle frequency (hence sweep rate) [16]. The hysteresis loss is determined by both the 

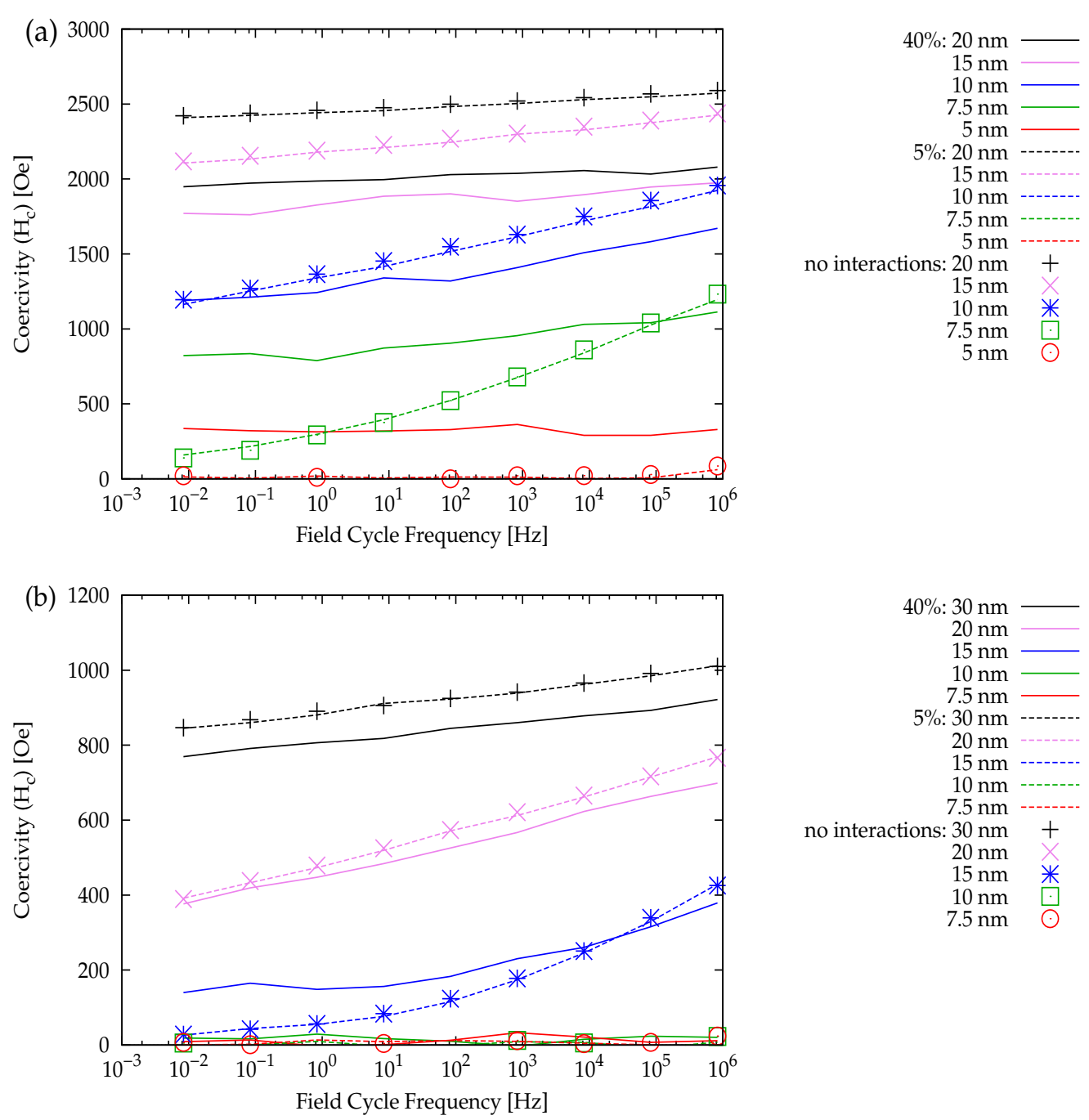

Figure 2: Field cycle frequency dependence of the coercivity for the composite systems consisting of (a) FePt particles between 5 and $20 \mathrm{~nm}$ and (b) magnetite particles between 7.5 and 30nm at packing densities of $5 \%$ and $40 \%$, thereby demonstrating the effects of the interparticle interactions. Note that larger magnetite particles are used in order to magnify the interaction effects in this weakly interacting system.

coercivity $H_{c}$ and the remanent magnetisation $M_{r}$, hence we initially concentrate on the effect of the interactions and field cycle frequency on these parameters.

The effect of the interactions on the field cycle frequency dependence of $H_{c}$ is shown in Fig. 2 for various particle sizes and for packing densities of $5 \%$ and $40 \%$. The field cycle frequency dependence for the fully non-interacting cases are also shown. The particle density is represented by a volume fraction, i.e., (magnetic volume)/(total volume), expressed as a percentage, and the non-interacting case was realised by setting the interaction field contribution to zero.

Fig. 2 shows the overall effects to be rather complex. The FePt composite system with a packing density as low as $5 \%$ and the magnetite composite systems, which have a 

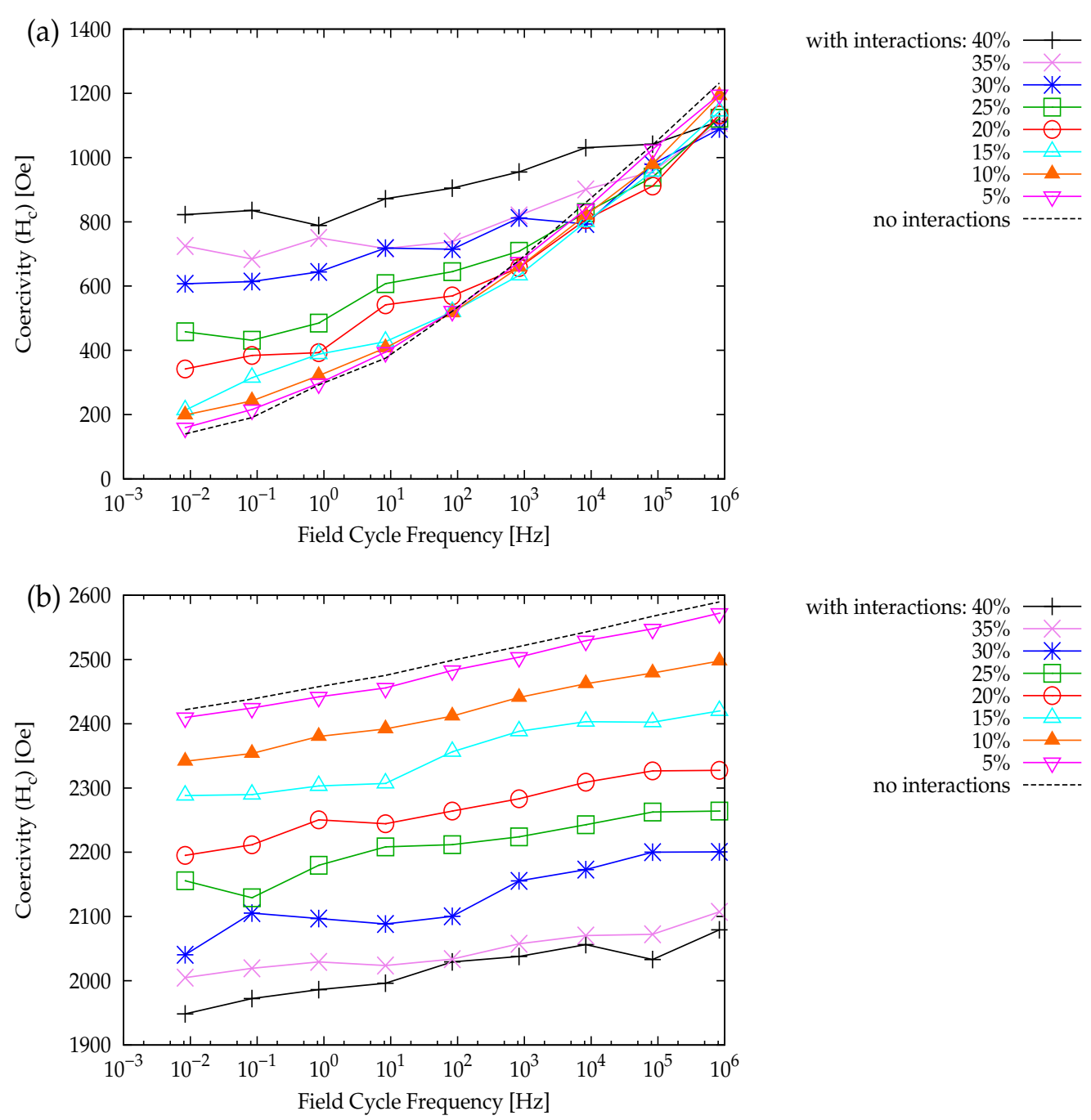

Figure 3: Field cycle frequency dependence of the coercivity for FePt particles of particle sizes (a) $7.5 \mathrm{~nm}$ and (b) $20 \mathrm{~nm}$ as a function of the packing density, demonstrating the different effects of interparticle interactions for low and high $H_{c}$ systems.

low saturation magnetisation, can in most cases be considered as non-interacting. The effect of the interactions on $H_{c}$ is seen to strongly depend upon the particle diameter. With respect to the FePt particles, the smallest diameter particles considered here ( $5 \mathrm{~nm})$ are clearly SPM in the absence of interactions. At a packing density of $40 \%$, however, the $5 \mathrm{~nm}$ FePt system develops a significant coercivity (and, hence, energy loss) due to the magnetostatic interactions. Conversely, larger FePt particles of $20 \mathrm{~nm}$ diameter exhibit a decrease of $H_{c}$ and therefore a reduction in overall energy loss as a consequence of the interactions.

To understand further the effect of the interactions, we consider in detail the behaviour of the FePt particles where the magnetostatic interaction is strong. Fig. 3 shows the field cycle frequency dependence of $H_{c}$ for FePt particles with diameters of $7.5 \mathrm{~nm}\left(\right.$ low $H_{c}$ ) and 20nm (high $H_{c}$ ) as a function of the packing fraction, $\epsilon$. The effects 
of the interactions is seen to be dramatically different in both of these cases. For the low diameter case at low field cycle frequencies (Fig. 3(a)), $H_{c}$ is seen to increase as a function of $\epsilon$, whereas for the 20nm diameter particles (Fig. 3(b)), $H_{c}$ decreases as a function of increasing $\epsilon$. The higher $H_{c}(20 \mathrm{~nm})$ system has a high remanence, which would tend to result in a demagnetising effect, thereby lowering the coercivity. In the absence of interactions, the $7.5 \mathrm{~nm}$ sample has close to zero remanence at low field cycle frequencies. In this case, the interactions appear to increase the $H_{c}$ value, which is consistent with the expected increase in energy barrier that is predicted by the model of Dormann et al. [24]. Thus, for low coercivity (close to SPM) systems, the prediction of increased energy barrier is borne out by our hysteresis calculations, with the situation being rather more complex for higher coercivity systems.

To model the coercivity as a function of the field cycle frequency, we start by considering a simple model in which the anisotropy easy-axes of the particles are aligned. The magnetisation of the system can be modelled by taking into account a volume distribution, such that,

$$
M / M_{s}=\int_{0}^{V_{c}} L(V, H) f(V) d V-\int_{V_{c}}^{V_{c}(H)} f(V) d V+\int_{V_{c}(H)}^{\infty} f(V) d V,
$$

where $L(V, H)$ is the Langevin function and $f(V)$ is the volume distribution function. The first integral on the right-hand-side of this equation represents the SPM particles, and the second and third integrals represent the reversed and non-reversed fractions of the thermally stable particles, respectively. $V_{c}$ and $V_{c}(H)$ are the critical volumes in a zero-field and in an applied field $H$, respectively.

In Ref. [21] it is shown that as long as the SPM fraction is negligible, the coercivity can be given by the so-called Sharrock law [22], where,

$$
H_{c}=H_{k}\left[1-\left(\frac{k_{B} T \ln \left(t f_{0}\right)}{K V_{m}}\right)^{1 / 2}\right],
$$

and $V_{m}$ is the median volume of the distribution. In our case, a comparison with Eq. 6 must be used with care since the systems in general are comprised of a mixture of SPM and thermally stable particles. Consequently, we might expect that eq. 6 will only be applicable for systems in which the particle size and size distribution have values which give rise to a small SPM fraction.

In order to better understand the effect of interactions, we have fitted the Sharrock law (eq. 6) to our computational results, with $f_{0}$ fixed at $10^{9} \mathrm{~s}^{-1}$. The aim of this fit is to consider the extent to which the interactions are reflected in the values of the main parameters, $\beta=K V / k_{B} T$ and $H_{K}$. Prior to the fitting, however, some modification is required since eq. 6 is derived under the assumption of a time-dependent magnetisation in a static field, whereas the calculations presented here assume a swept field.

It has previously been shown that the stepped field and swept field processes are related by the expression,

$$
t_{\text {eff }}=\frac{R^{-1} H_{K}}{2 \beta\left(1-H_{c} / H_{K}\right)},
$$



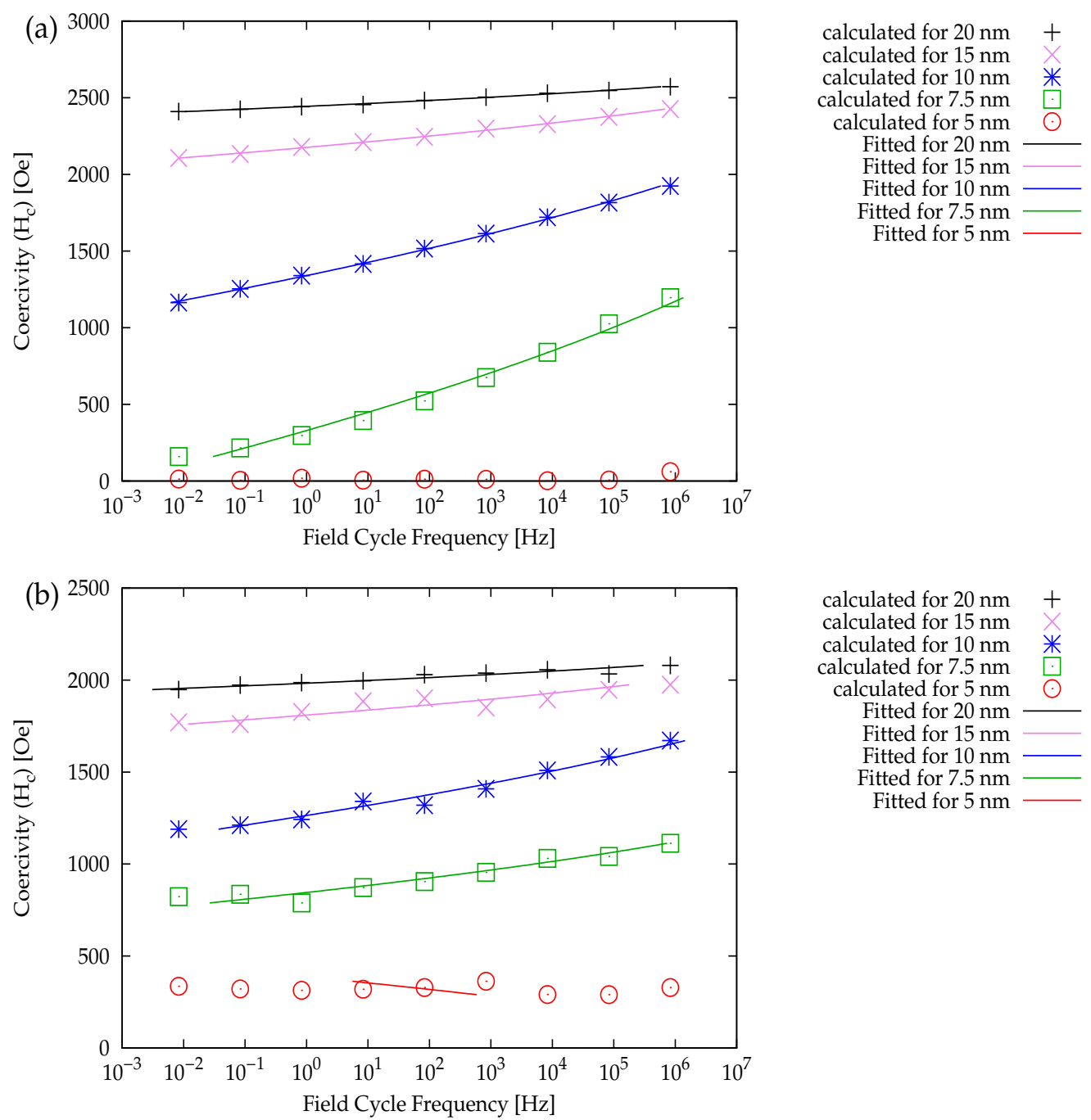

Figure 4: Fitting of the Sharrock law (eq. 6) to the coercivity as a function of the field cycle frequency for the FePt nanoparticle composite at packing densities of (a) $5 \%$ and (b) $40 \%$.

where $t_{\text {eff }}$ is the effective time to be used in eq. 6 and $R$ is the field cycle frequency [23]. The fit of eq. 6 (with $t$ replaced by $t_{e f f}$ ) to the computational results is carried out using a Levenberg-Marquardt algorithm, which minimises the least squares deviation between the modified Sharrock law and the calculated $H_{c}$ values.

The results of the fitting procedure (Fig. 4) indicate that eq. 6 provides a good representation of the field cycle frequency dependence of $H_{c}$ within the constraint of predominantly SPM behaviour. Such a good comparison is perhaps surprising, since the Sharrock law is derived for a system of aligned particles, whereas our calculations assume a 3-D random dispersion of easy-axis directions. The random alignment of the easy-axes are expected to affect the exponent of $1 / 2$ in eq. 6 , with Victora suggesting that an exponent of $2 / 3$ as being more appropriate [25]. It would seem, however, that the fit that we have achieved is not sensitive to the exponent. Interestingly, the fit to the 

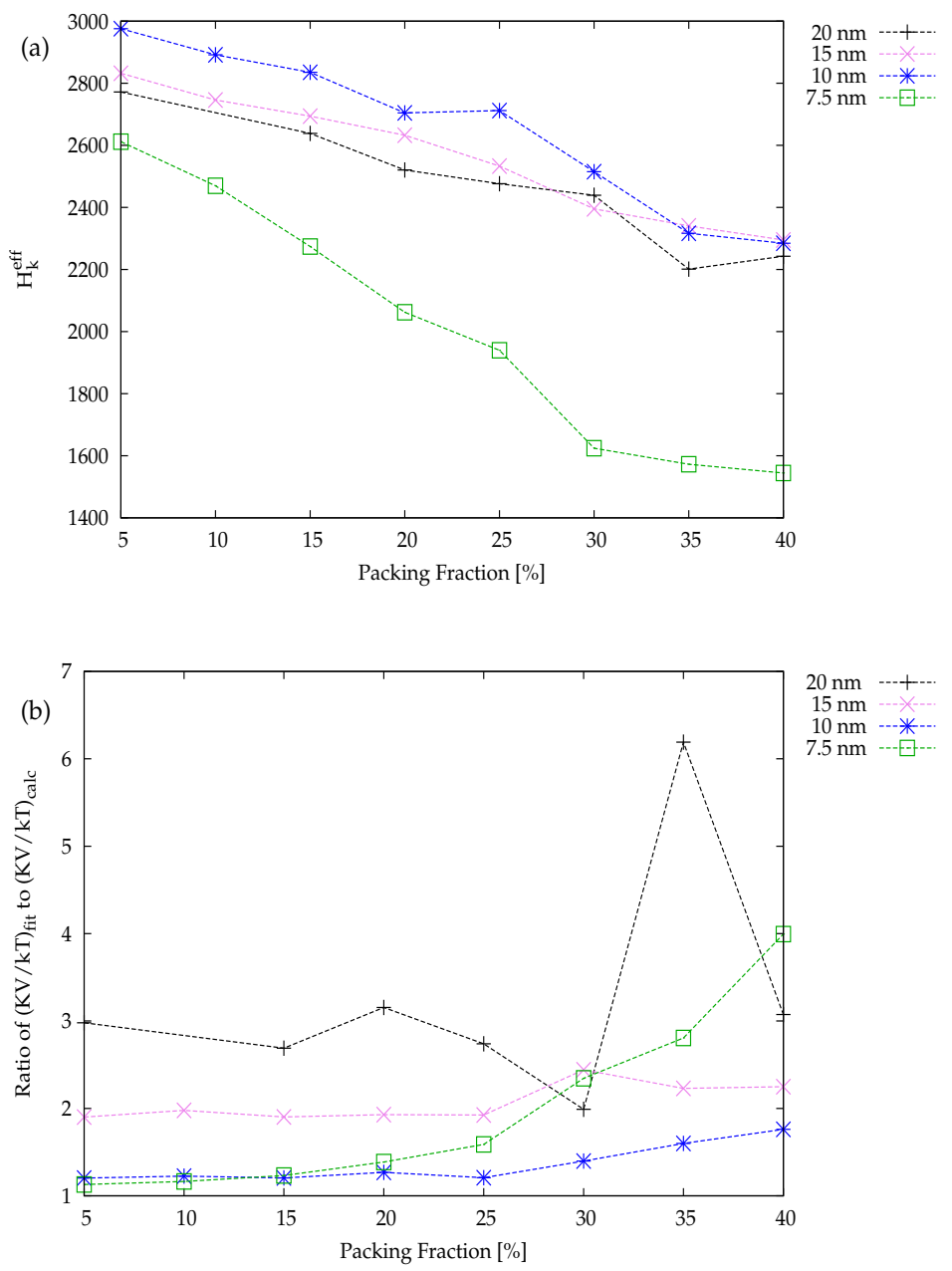

Figure 5: The fit parameters (a) $H_{K}^{\text {eff }}$ and the effective value of (b) $K V / k_{B} T\left(\beta^{\text {eff }}\right)$ for the $\mathrm{FePt}$ particle composite as a function of packing density.

Sharrock law is also acceptable at the higher packing density of $40 \%$, except for the case of the lowest diameter, where we recall that the coercivity is determined predominantly by the interparticle interactions.

The fit to eq. 6 gives rise to fitting parameters, which are found to be dependent on the interparticle interactions. Figs. 5(a) and (b) show the effective parameters $H_{K}^{e f f}$ and $K V / k_{B} T\left(\beta^{e f f}\right)$ as a function of the packing fraction, respectively. In the case of $\beta^{e f f}$, each value is normalised with respect to the value for the non-interacting case. The fitting parameters demonstrate an interesting dependence on the packing fraction. Specifically, $H_{K}^{e f f}$, which represents the intrinsic coercivity, i.e., in the absence of thermal effects, is seen to decrease monotonically with increasing packing fraction. For the larger particles there also appears to be no significant dependence of $H_{K}^{e f f}$ on the particle size. A complex and subtle dependence of the parameter $\beta^{e f f}$, which represents the degree of enhancement of the thermal stability on the packing density and particle size, also 
exists (Fig. 5(b)). Overall, there is an enhancement of $K V / k_{B} T\left(\beta^{\text {eff }}>1\right)$ due to the interactions, which increases with increasing particle diameter. This effect is particularly noticable for the particle diameter of $7.5 \mathrm{~nm}$, which shows a pronounced increase in $\beta^{e f f}$ as a function of the packing fraction. These trends suggest that the enhancement of the energy barrier is perhaps greatest for particles close to the SPM limit.

Overall, we can conclude that the effects of interactions on the energy barrier are complex, but can be represented in terms of an effective energy barrier of the form,

$$
E_{b}=\left(\frac{K V}{k_{B} T}\right)^{e f f}\left[1-\frac{H}{H_{K}^{e f f}}\right]^{2} .
$$

Clearly one cannot necessarily state that the energy barrier is increased or decreased by interactions. Instead, the effect of interactions on the energy barrier and coercivity is dependent on the relative values of effective stability and intrinsic coercivity, which are determined by the packing density and particle size. In the following, we investigate the effect of interactions on the net hysteresis loss of the system, concentrating on the variation of the energy loss as a function of the field cycle frequency.

\subsection{Field cycle frequency dependence of the energy loss}

We commence this investigation by considering the energy loss per hysteresis cycle. To emphasise the dual role of the packing density, $\epsilon$, we write $E=\epsilon M_{s} \int M_{\text {red }} d H$ where the integral is taken over a single cycle. In this equation, $M_{s}$ is the saturation magnetisation and $M_{\text {red }}$ is the reduced magnetisation (relative to the saturation magnetisation $\epsilon M_{s}$ ) of the system at a given packing density. Clearly the effect of increasing the packing density is a complex convolution of the increase due to the increased magnetic fraction with the effects of the interactions on $M_{\text {red }}$ - the latter being essentially determined through the parameters $H_{K}^{e f f}$ and $\beta^{\text {eff }}$.

The energy losses per cycle at a field cycle frequency of $\sim 100 \mathrm{~Hz}$ are shown in Fig. 6 for (a) FePt and (b) magnetite as a function of the packing density and the particle size. The difference in magnitude of the energy loss between the materials is predominantly due to the difference between their saturation magnetisation values. For magnetite, this loss, is essentially linear with the packing density, as would be expected for a non-interacting or weakly interacting dipolar system. At high packing densities, the interactions in this system have only a minimal effect. In the case of FePt, the comparison is more complex, with significant deviations from linearity that occur due to the strong interactions. Enhancement of the energy barrier results in an increase in the energy loss for small FePt particle sizes, whereas the reduction in $H_{K}^{e f f}$ dominates for large sizes leading to a reduction in the energy loss.

The effect of interactions on the field cycle frequency dependence of the energy loss/cycle is shown in Fig. 7 for FePt particle sizes of (a) $7.5 \mathrm{~nm}$ and (b) 20nm. The enhancement of the loss/cycle due to increasing interactions (i.e., at higher packing densities) for the smaller diameter system is pronounced at low field cycle frequencies. However, the enhanced thermal stability factor $\beta^{\text {eff }}$ and reduced $H_{K}^{\text {eff }}$ resulting from 

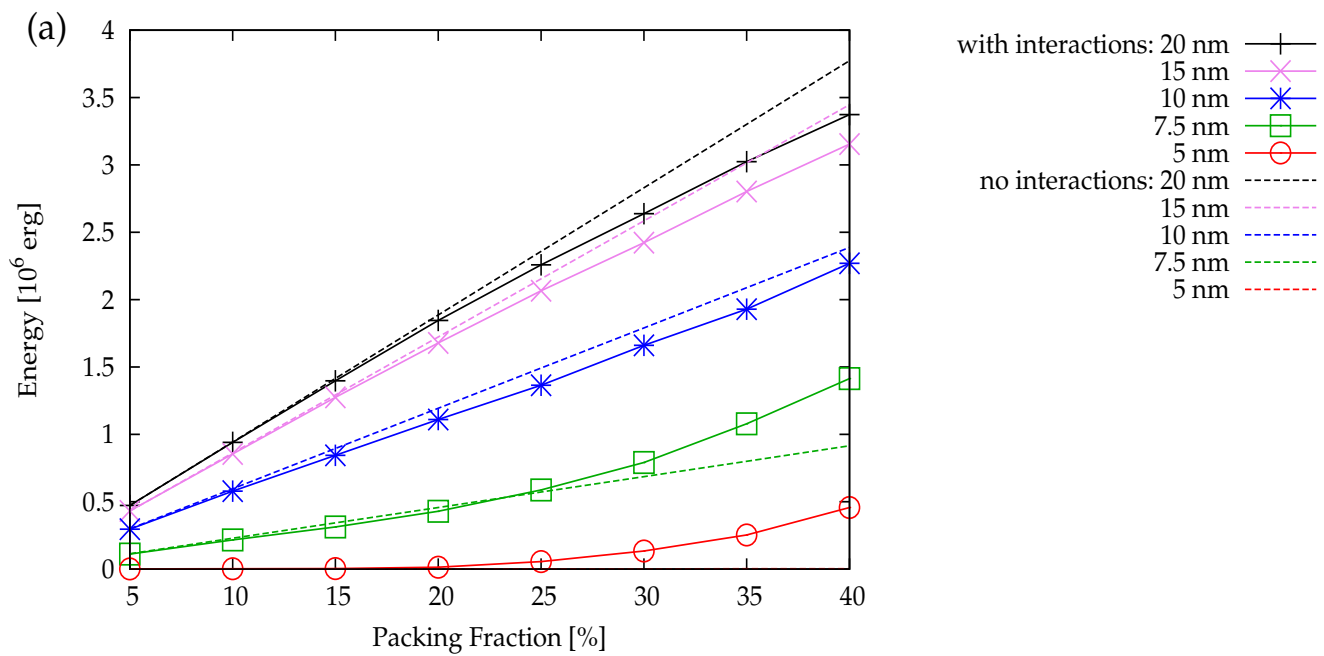

(b)
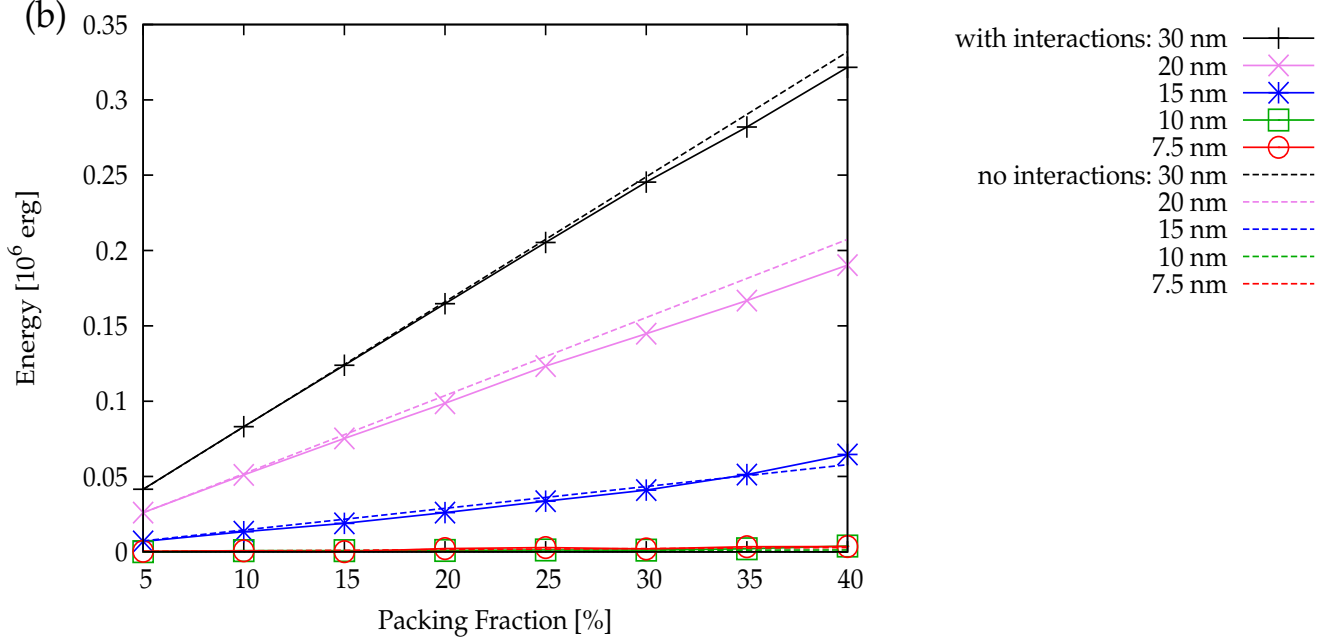

Figure 6: Energy loss per cycle at a field cycle frequency of $\sim 100 \mathrm{~Hz}$ for (a) FePt showing non-linearities due to strong interactions, and (b) magnetite having linear behaviour as a function of the packing density and particle size.

the interactions lead to a much weaker variation of the energy loss/cycle as a function of the increasing field cycle frequency. In the case of the larger diameter systems, the predominant effect is in the reduction in $H_{K}^{e f f}$ with increasing interactions (i.e., packing densities), leading to a reduction in the loss/cycle due to interaction effects.

Finally, we investigate the most important factor for hyperthermia applications, which is the rate of hysteresis loss, i.e., $R=f \epsilon M_{s} \int M_{\text {red }} d H$, where $f$ is the frequency of the applied field. This factor is responsible for the degree of magnetic heating. As the frequency increases, the field which can be brought to bear is reduced, not only for the physiological reasons mentioned earlier, but also due to the physical difficulty of producing high fields over large volumes at high frequencies. As a result, the magnetic systems at high frequency are likely to be subjected to non-saturating magnetic fields and will therefore be cycled around minor hysteresis loops with a consequent reduction 

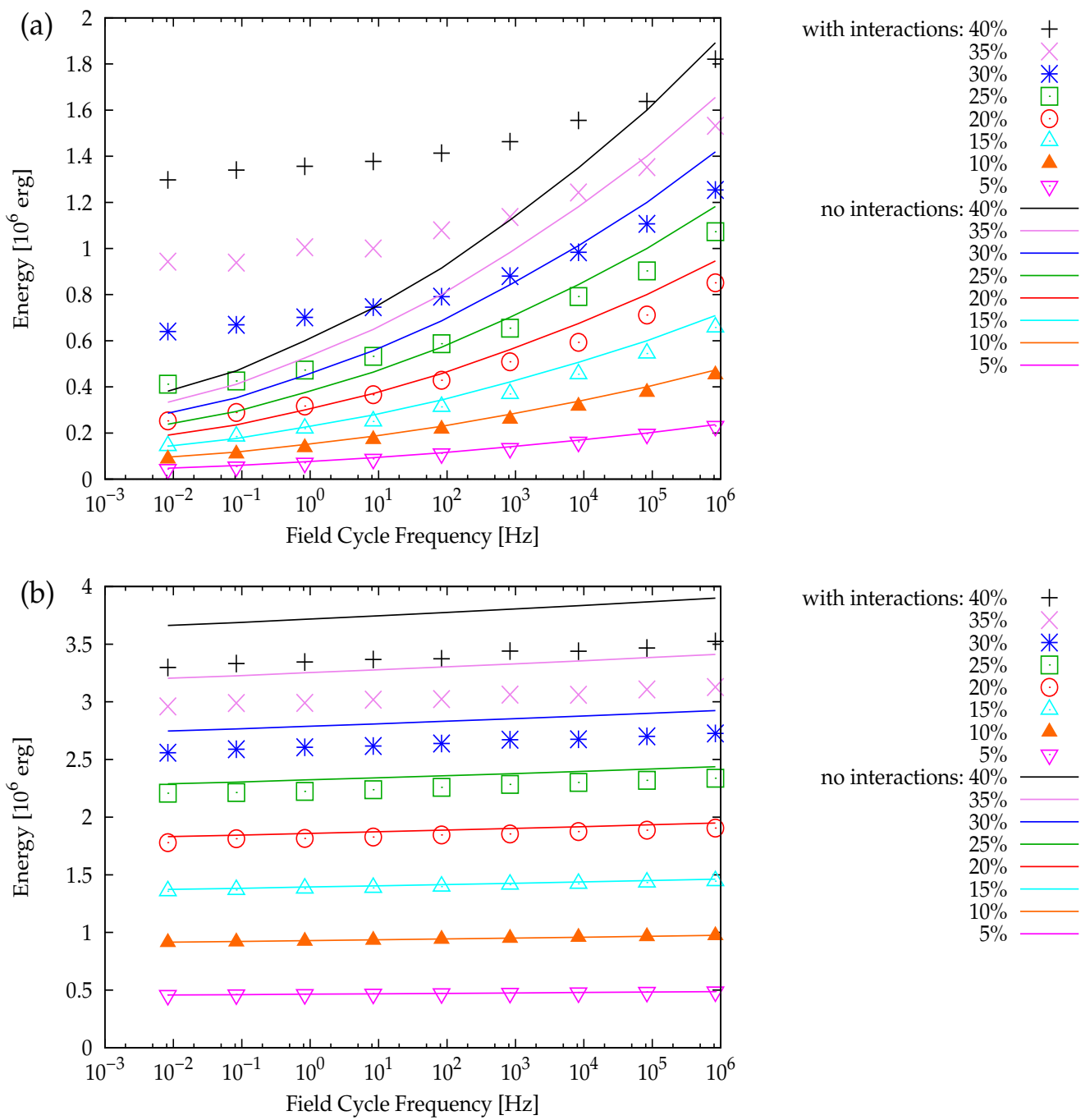

Figure 7: Energy loss per cycle as a function of the field cycle frequency for FePt particles of diameters (a) $7.5 \mathrm{~nm}$ and (b) FePt 20nm for various packing fractions.

in the hysteresis loss.

In order to generate minor loops, the field must be applied to an initially demagnetised sample. For a strongly interacting system, a demagnetised (ground) state cannot be produced by a random assignment of the initial moment direction of the particles since this would produce a relatively high energy state. The magnetic groundstate was therefore first determined by a process of slow ac erasure, whereby fields of increasing frequency and decreasing magnitude were applied to demagnetise the system. The demagnetised system was then subjected to two field cycles to eliminate transient behaviour and to attain reproducible minor loops, with the third loop being stored for analysis.

Fig. 8 shows the power generated as a function of the frequency for FePt particles at packing fractions of $5 \%$ and $40 \%$, under the assumption that the maximum available 


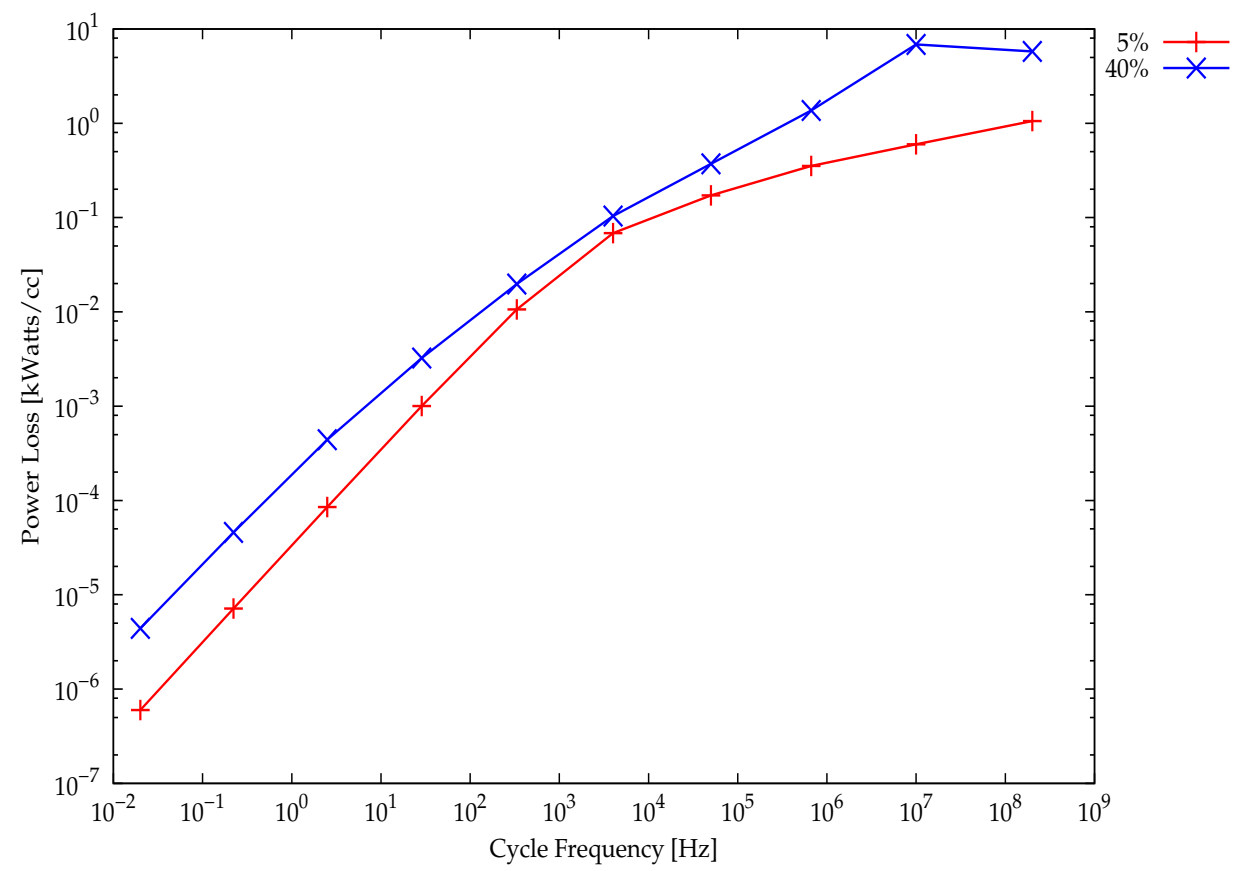

Figure 8: Rate of energy loss per cycle as a function of the cycle frequency for an FePt particle composite with packing densities of $5 \%$ and $40 \%$.

field falls off at $10 \%$ per decade. The power generated increases non-linearly due to the combined effect of the proportionality to frequency and the increased coercivity at high frequencies. At a packing density of $40 \%$ there is a very rapid increase in the power output at high frequencies, presumably due to the effect of interactions. We also note the appearance of a peak in the power output at high frequencies as the available field becomes insufficient to saturate the material. This may be due to the demagnetised state being very stable for a strongly interacting system. The slope $=$ 1 at low frequencies is a well known dependence of 'hysteresis' losses associated with short length scales at which Barkhausen jumps occur (in our case, the switchings of individual particles) [26]. In materials where eddy currents are present, for example, there can in addition be quadratic and higher order contributions associated with other relevant length scales in the system, such as the geometrical size of the system, domain size, etc., with the resulting losses being a superposition of the contributions from these different loss types. This remarkable observation is known as 'magnetic loss separation' $[26,27]$. In the composite systems that we have studied, we do not have eddy currents, however, there could still be some analogies. For example, in Fig. 8, the slopes change due to the interactions. Thus, it could be very interesting to study the 'loss separation' (if it exists) and reasons for it - a factor which will be important in further studies of these systems. 


\section{Conclusion}

We have developed a model of an interacting fine particle system, which takes into account the dipolar interaction between the particles. The model has been applied to the study of hysteresis losses as related to magnetically induced hyperthermia. Central to the investigation are calculations of the field cycle frequency dependence of the coercivity and the hysteresis (energy) loss. Firstly, the effects of interactions were investigated by fitting the Sharrock law to the variation of $H_{c}$ with field cycle frequency (hence sweep rate). The Sharrock law describes the data remarkably well, given that it was derived for a system of perfectly aligned, non-interacting particles. Importantly, however, we find that the fitting leads to effective parameters $H_{K}^{e f f}$ and $\beta^{e f f}=K V / k_{B} T$, which are themselves dependent on the interaction strength. The increase or decrease of $H_{c}$ with interactions depends upon the relative change of $H_{K}^{e f f}$ and $\beta^{\text {eff }}$.

The behaviour of the effective parameters demonstrates the complex effects of interactions. The increase of $\beta^{e f f}$ with packing density is certainly consistent with the model of Dormann et. al. [24], which predicts increased energy barriers arising from the dipolar interactions. This seems to be primarily responsible for an increase in the coercivity and thermal stability for systems that are superparamagnetic at low densities, and are also close to the SPM/thermally stable transition. The reduction of $H_{K}^{\text {eff }}$ with increasing interaction strength, however, acts to lower the coercivity. In systems with large particle size and consequently high $H_{c}$, this is the dominant effect leading to a reduction in $H_{c}$. The distillation of interaction effects into two simple parameters is clearly of interest and requires further investigation in order to determine the underlying physics of the relationships, especially in relation to the effects of temperature and the magnetic state of the system.

The interparticle interactions have a complex effect on the energy loss per cycle. The energy loss is reduced for larger particles since the reduction in coercivity dominates, along with a corresponding reduction in the remanence. For small particle sizes, the energy loss is enhanced. The dominant mechanism here seems to be an enhancement of the energy barrier due to interactions, which changes the nature of the particles from SPM to thermally stable. This effect might be expected to be strongly dependent on the particle size and also the size dispersion, since it relies on the presence of particles close to the SPM limit. As expected, the hysteresis loss increases with frequency as long as the applied field is sufficiently large to achieve magnetic saturation. At high frequencies, the available maximum field is reduced, thereby leading to a peak in the hysteresis loss at a given frequency for the strongly interacting FePt system.

\section{References}

[1] G.M. Hahn, Cancer Research 39, 2264 (1979).

[2] B. Hildebrandt, P. Wust, O. Ahlers, A. Dieing, G. Sreenivasa, T. Kerner, R. Felix and H. Reiss, Critical Reviews in Oncology/Hematology 43, 33 (2002). 
[3] Q.A. Pankhurst, N.K.T. Thanh, S.K. Jones, and J. Dobson, J. Phys. D: Appl. Phys. 42, 224001 (2009).

[4] Q.A. Pankhurst, J. Connolly, S. K. Jones, and J. Dobson, J. Phys. D: Appl. Phys. 36, R167R181, (2003).

[5] A. Jordan, R. Scholz, K. Maier-Hauff, M. Johannsen, P. Wurst, J. Nadobny, H. Schirra, H. Schmidt, S. Deger, S. Loening, W. Lanksch and R. Felix, Journal of Magnetism and Magnetic Materials 225, 118 (2001).

[6] R.E. Rosensweig, Journal of Magnetism and Magnetic Materials 252, 370 (2002).

[7] R. Hergt, S. Dutz and M. Roder, J Phys. Condens. Matter, 20, 385214 (2008).

[8] R. Ramprasad, P. Zurcher, M. Petras, M. Miller and P. Renaud, Journal of Applied Physics 96, 519 (2004).

[9] J. Sun, S. Zhou, P. Hou, Y. Yang, J. Weng, X. Li, and M. Li, Journal of Biomedical Materials Research Part A 80, 333 (2007).

[10] T.L. Kline, Y-H. Xu, Y. Jing, and J-P. Wang, Journal of Magnetism and Magnetic Materials 321, 1525 (2009).

[11] P. de la Presa, T. Rueda, M.P. Morales and A. Hernando, IEEE Transactions on Magnetics, 44, 2816 (2008).

[12] R.W. Chantrell, N.S. Walmsley, J. Gore and M. Maylin, Phys. Rev. B. 63, 24410 (2001).

[13] A.G. Roca, M.P. Morales and C.J. Serna, IEEE Transactions on Magnetics 42, 3025 (2006).

[14] X.W. Wu, K.Y. Guslienko, R.W. Chantrell, and D. Weller, Applied Physics Letters 823475 (2003).

[15] P. Guardia, B. Batlle-Brugal, A.G. Roca, O. Iglesias, M.P. Morales, C.J. Serna, A. Labarta and X. Batlle, Journal of Magnetism and Magnetic Materials, 316, e756 (2007).

[16] E.C. Stoner and P. Wohlfarth, Phil. Trans. R. Soc. London, Ser. A 240, 599(1948).

[17] L Néel Ann Geophys (C.N.R.S) 5, 99 (1949).

[18] H. Pfeiffer, Phys. Status Solidi A 118,295 (1990).

[19] Metropolis et al., J. Appl. Phys. 21, 1087(1953).

[20] J. M. Qiu and J. P. Wang, Adv. Mater. (Weinheim, Ger.) 19, 1703 (2007).

[21] R.W. Chantrell, D. Weller, T.J. Klemmer, S. Sun, E.E.Fullerton J. Appl. Phys. 91, 6866 (2002).

[22] M. P. Sharrock, IEEE Trans. Magn. 26, 193 (1990).

[23] R.W. Chantrell, G.N. Coverdale and K. O'Grady J. Phys. D. 211469 (1988).

[24] J.L. Dormann, D. Fiorani, and E. Tronc, Adv. Chem. Phys. XCVIII, 283 (1997).

[25] R.A. Victora, Phys. Rev. Lett. 63, 457 (1989).

[26] G. Bertotti, 'Hysteresis in Magnetism' (Academic, San Diego, 1998), p. 24.

[27] F. Colaiori, G. Durin, S. Zapperi, Phys. Rev. Lett 97, 257203 (2006). 